\title{
Insights into Intermolecular Interactions of Spironolactone Solvates
}

Lihong Jia ${ }^{a}$, Shihao Zhang ${ }^{a}$, Wenchao Yang ${ }^{a}$, Ying Bao ${ }^{a, b, c}$, Baohong Hou ${ }^{a, b, c}$, Ling Zhou $^{a^{*}}$ and Qiuxiang Yin ${ }^{a, b, c^{*}}$

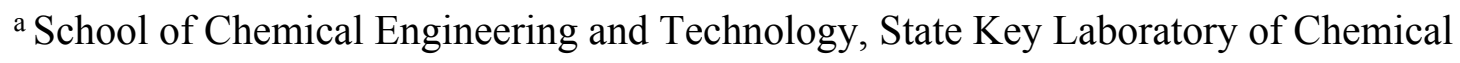
Engineering, Tianjin University, Tianjin 300072, People's Republic of China.

${ }^{\mathrm{b}}$ Co-Innovation Center of Chemistry and Chemical Engineering, Tianjin University, Tianjin 300072, People's Republic of China.

c Tianjin Key Laboratory of Modern Drug Delivery and High Efficiency, Tianjin 300072, People's Republic of China.

\section{Supporting Information}

Table S1. SPI crystal forms obtained from recrystallization in different solvents.

\begin{tabular}{ccc}
\hline Solvent & Crystallization method & Obtained forms \\
\hline Mixture of water and acetone (1:4) & Cooling/slurry/evaporation & Hydrate \\
Methanol & Cooling/slurry/evaporation & $\mathrm{S}_{\mathrm{MeOH}}$ \\
Ethanol & Cooling/slurry/evaporation & $\mathrm{S}_{\mathrm{EtOH}}$ or Form II or mixture \\
n-Propanol & Cooling/slurry/evaporation & Mixture of $\mathrm{S}_{\mathrm{NPA}}$ and Form II \\
i-Propanol & Cooling/slurry/evaporation & Mixture of $\mathrm{S}_{\mathrm{IPA}}$ and Form II \\
Acetonitrile & Evaporation & $\mathrm{S}_{\mathrm{ACN}-\mathrm{new}}$ \\
Formic acid & Slurry/evaporation & $\mathrm{S}_{\mathrm{FA}}$ \\
Acetic Acid & Slurry/evaporation & $\mathrm{S}_{\mathrm{AA}}$ \\
Benzene & Slurry/evaporation & $\mathrm{S}_{\mathrm{Ben}}$ \\
Toluene & Slurry/evaporation & $\mathrm{S}_{\mathrm{Tol}}$ \\
Acetone & Cooling/slurry/evaporation & Form II \\
\hline
\end{tabular}



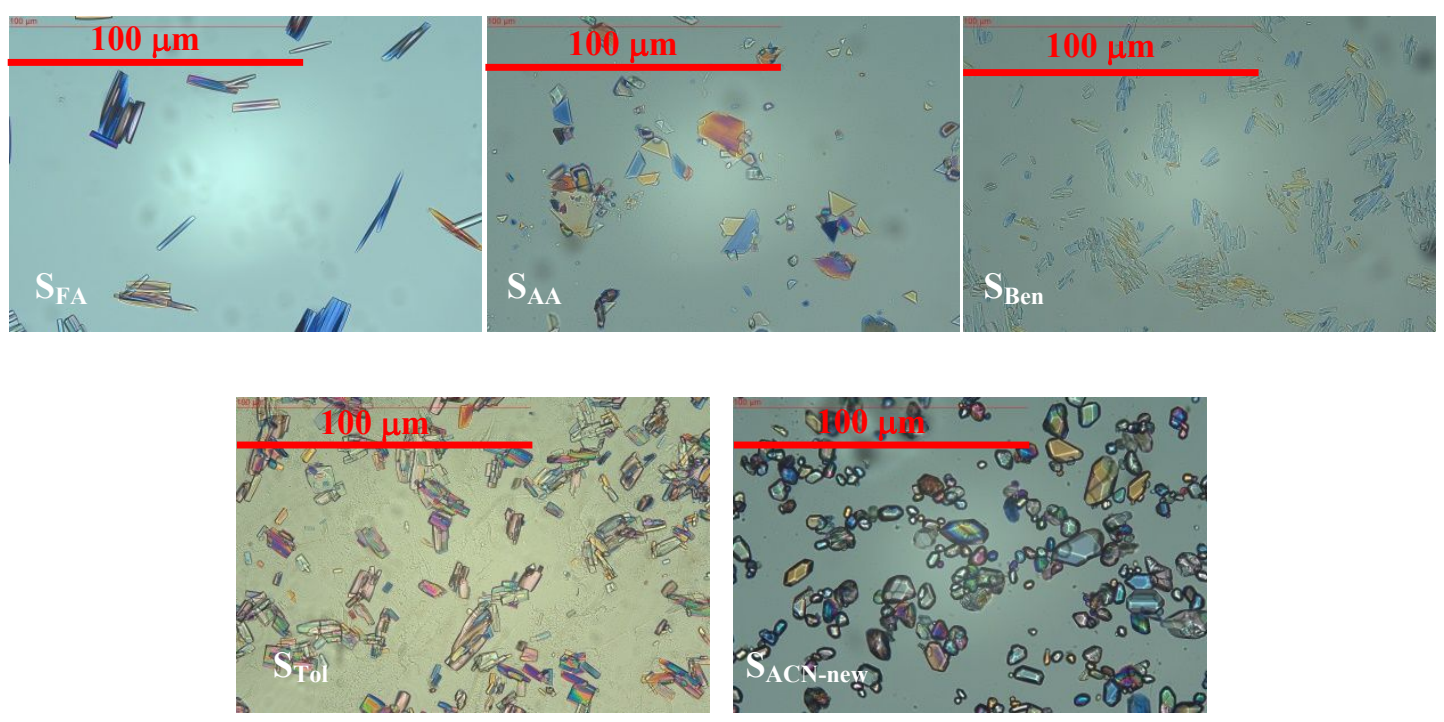

Figure S1. Polarizing microscope (PLM) images of SPI solvates (from cooling crystallization)

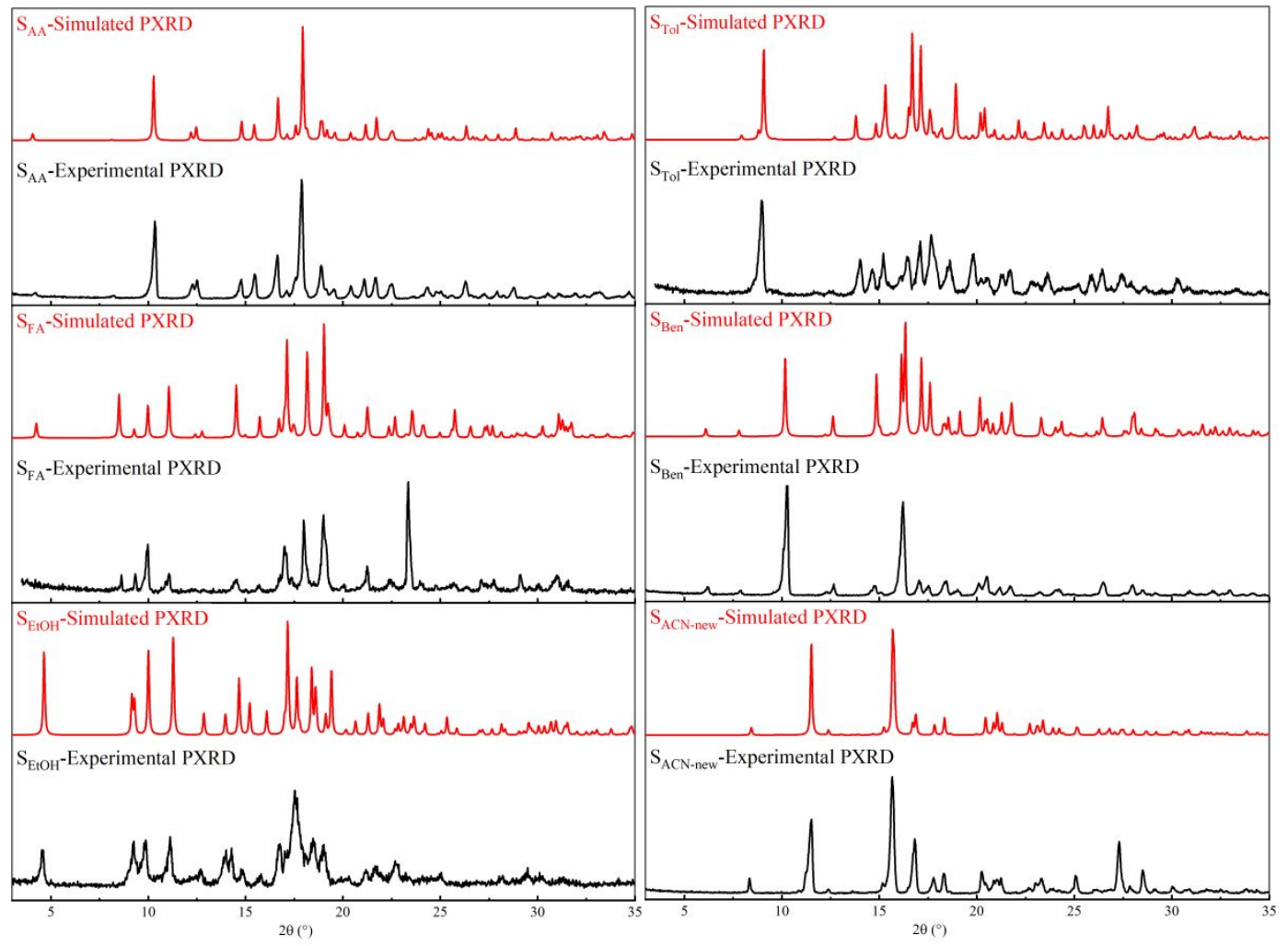

Figure S2. The PXRD patterns of experimental and simulated from the single crystal. 


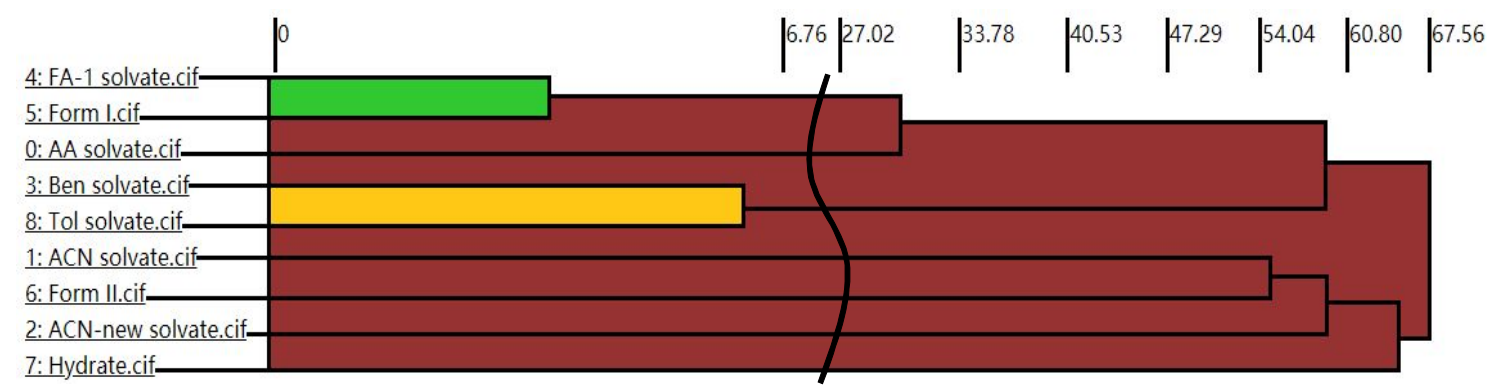

Figure S3. Packing similarity tree diagram of SPI forms. The horizontal axis corresponds to the

PSab value (similarity). Dark green indicates almost identical packing and dark red indicates dissimilar packing. The part of dendrogram, between 7 and 26 was removed to make the figure shorter and more readable. A cluster size of 15 SPI molecules was used.

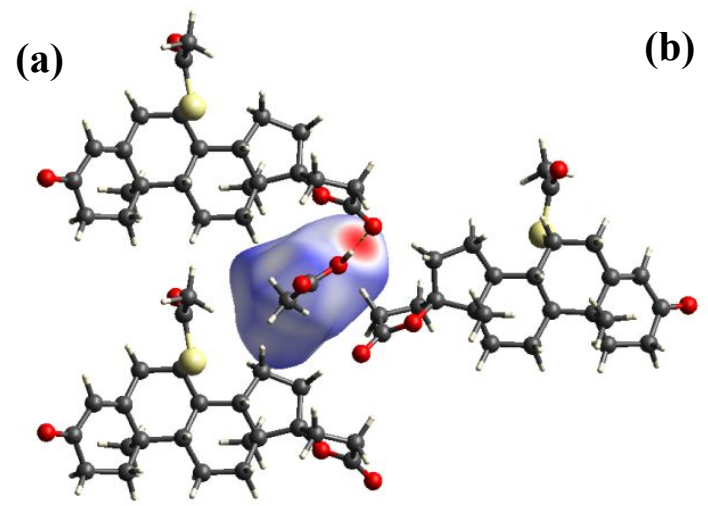

(b)

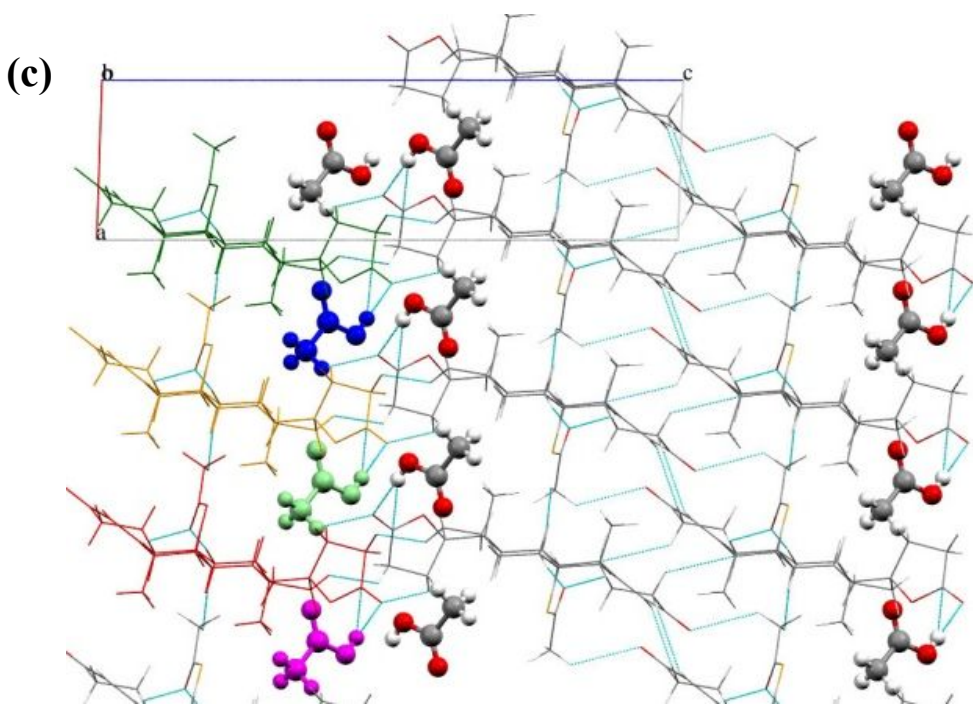




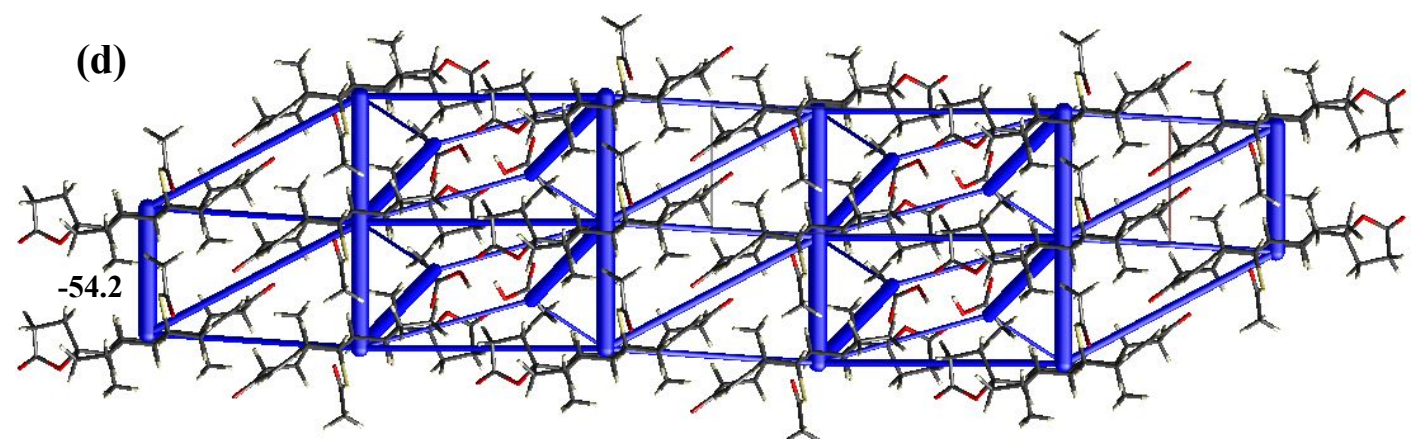

Figure S4. (a) $d_{\text {norm }}$ plot of the solvent molecule mapped on the surface. (b) Hirshfeld surface of the solvent molecule. (c) Packing diagram of $\mathrm{S}_{\mathrm{AA}}$ viewed along b axis; (d) Energy frameworks (total energy) for $\mathrm{S}_{\mathrm{AA}}$. The energy scale factor is 80 ; interaction energies with magnitudes higher than $-10 \mathrm{~kJ} / \mathrm{mol}$ have been omitted.

(a)

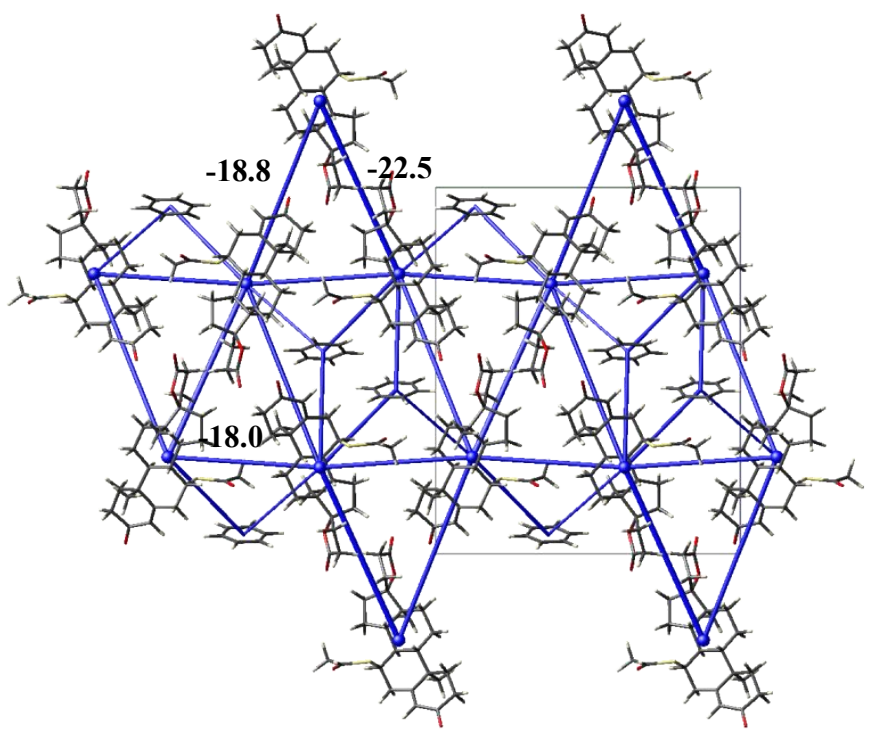

(b)

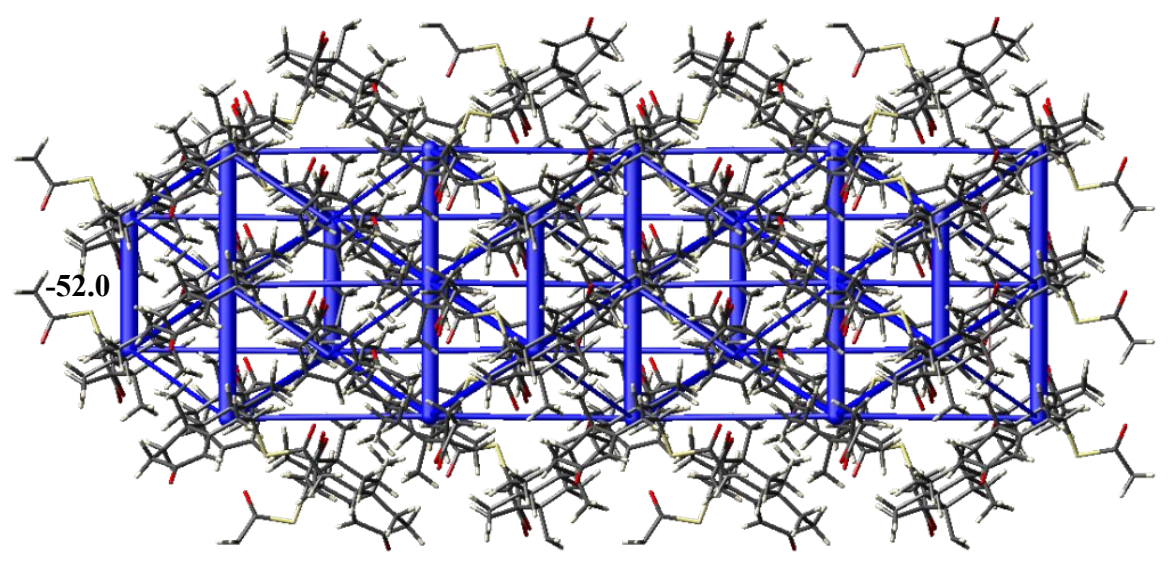


(c)

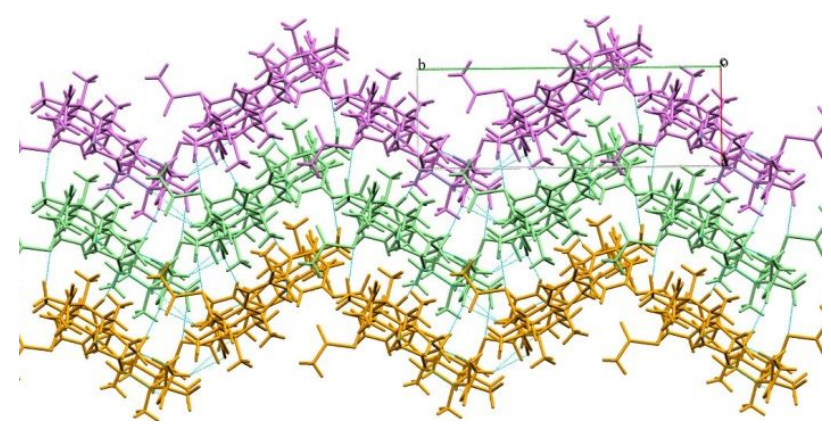

Figure S5. (a) Energy frameworks of $\mathrm{S}_{\mathrm{Ben}}$ viewed along a axis; (b) Energy frameworks of $\mathrm{S}_{\mathrm{Ben}}$ viewed along c axis. The energy scale factor is 80 ; interaction energies with magnitudes higher than $-10 \mathrm{~kJ} / \mathrm{mol}$ have been omitted; (c) Packing in $\mathrm{S}_{\mathrm{Ben}}$ viewed along the $\mathrm{c}$ axis, different colors represent different chains.

(c)
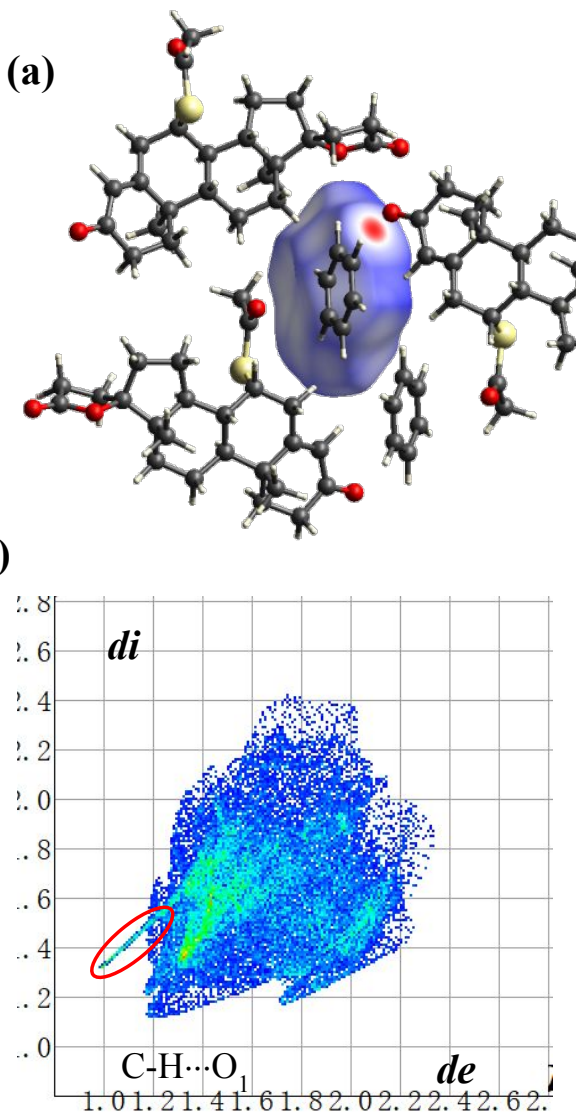

(b)

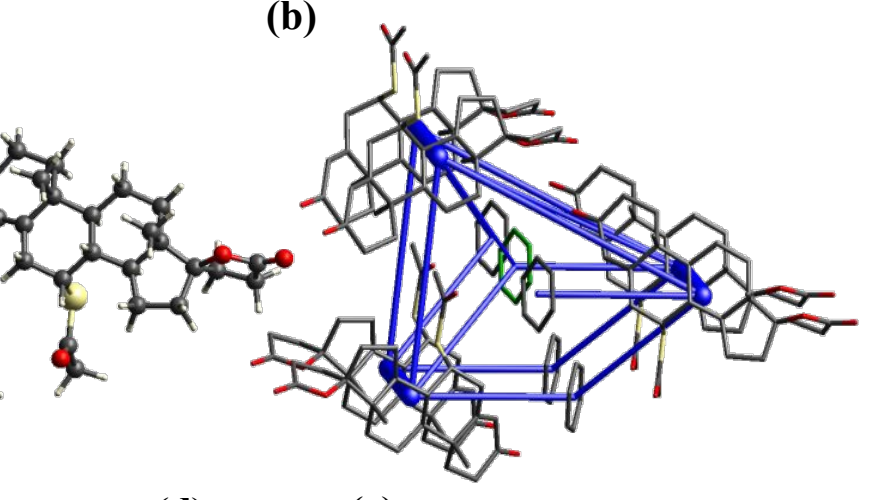

(d)

(e)

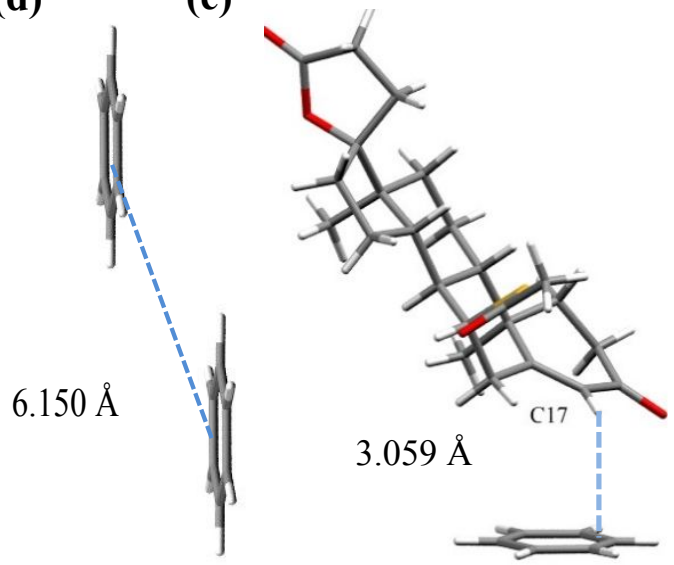

Figure S6. (a) Hirshfeld surface of the solvent molecule; (b) Energy framework for the solvent (highlighted in green) and adjacent molecules in $\mathrm{S}_{\text {Ben }}$ (cut-off radius $3.8 \AA$ ); (c) $\mathrm{d}_{\text {norm }}$ plot of the solvent molecule mapped on the surface; (d) The distance of two benzene molecules in the channel; (e) $\mathrm{C}-\mathrm{H} \cdots \pi$ interaction. 
(a)

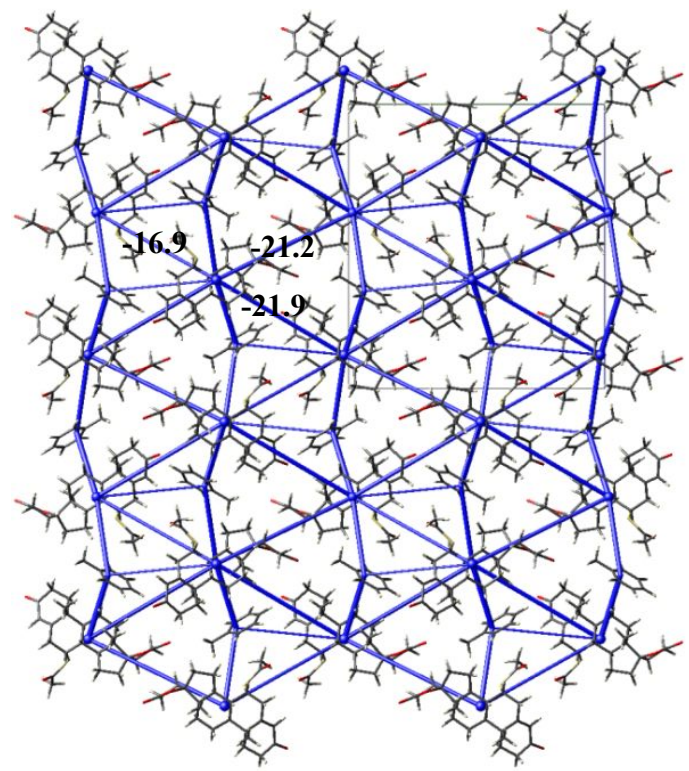

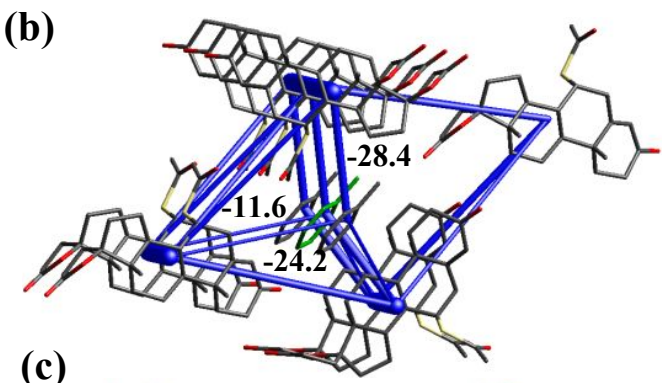

(c)

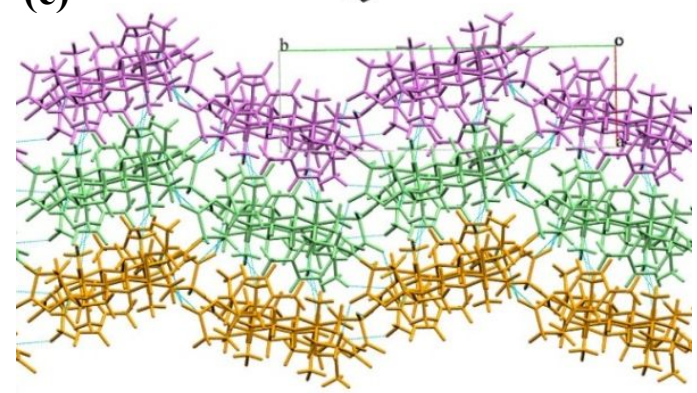

(d)

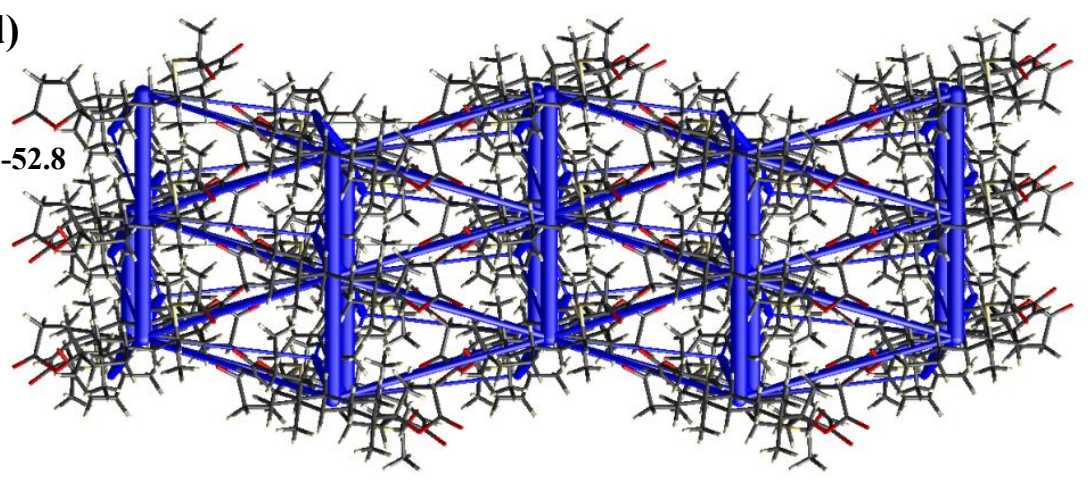

Figure S7. (a) Energy frameworks of $\mathrm{S}_{\mathrm{Tol}}$ viewed along a axis; (b) Energy framework for the solvent (highlighted in green) and adjacent molecules in $\mathrm{S}_{\text {Tol }}$ (cut-off radius $3.8 \AA$ ); (c) Packing in $\mathrm{S}_{\mathrm{Tol}}$ viewed along the $\mathrm{c}$ axis, different colors represent different chains. (d) Energy frameworks of $\mathrm{S}_{\mathrm{Tol}}$ viewed along $\mathrm{c}$ axis. The energy scale factor is 80 ; interaction energies with magnitudes higher than $-10 \mathrm{~kJ} / \mathrm{mol}$ have been omitted.

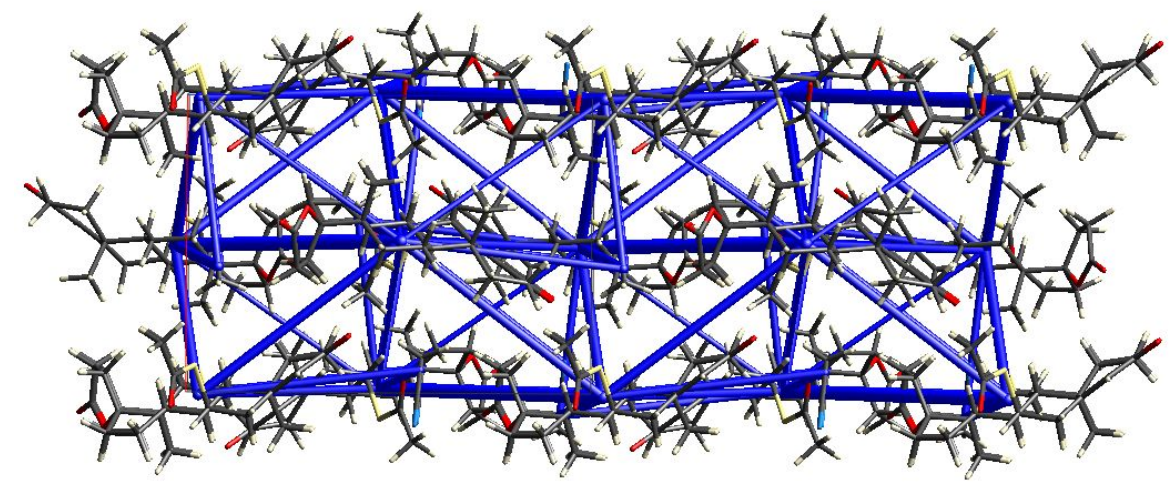

Figure $\mathrm{S} 8$. Energy frameworks (total energy) for $\mathrm{S}_{\mathrm{ACN}-\mathrm{new}}$. The energy scale factor is 80 ; interaction energies with magnitudes higher than $-10 \mathrm{~kJ} /$ mol have been omitted. 
Table S2 Pair-wise intermolecular interaction energies of SPI forms

\begin{tabular}{|c|c|c|c|c|c|c|c|c|}
\hline Interaction & Molecules pairs & $\begin{array}{c}\mathrm{R}_{\text {dist }} / \\
\AA\end{array}$ & $E_{\text {ele }}$ & $E_{\mathrm{pol}}$ & $E_{\mathrm{dis}}$ & $E_{\text {rep }}$ & $E_{\text {tot1 }}$ & $\begin{array}{c}E_{\text {tot2 }} \\
\text { (Gaussian) }\end{array}$ \\
\hline \multicolumn{9}{|c|}{$\mathrm{S}_{\mathrm{FA}}$} \\
\hline $1(2)$ & SPI $\cdots$ SPI & 6.16 & -13.7 & -5.6 & -63.4 & 33.7 & -53.0 & -53.9 \\
\hline $2(1)$ & SPI $\cdots$ FA & 8.69 & -78.1 & -18.2 & -9.3 & 94.8 & -45.6 & -49.5 \\
\hline $3(2)$ & SPI $\cdots$ SPI & 11.11 & -7.3 & -3.6 & -22.7 & 13.4 & -21.9 & -24.9 \\
\hline $4(2)$ & SPI $\cdots$ SPI & 12.63 & -9.6 & -4.3 & -19.4 & 15.5 & -20.6 & I \\
\hline $5(1)$ & SPI $\cdots$ FA & 5.43 & -9.1 & -8 & -21 & 21.7 & -20.5 & l \\
\hline $6(2)$ & FA $\cdots$ FA & 3.68 & -19.3 & -2.6 & -6.6 & 14.3 & -19.2 & / \\
\hline $7(2)$ & SPI $\cdots$ SPI & 9.78 & -5.8 & -2.7 & -17.6 & 9.5 & -17.5 & 1 \\
\hline $8(1)$ & SPI $\cdots F A$ & 6.43 & -10.3 & -2.4 & -19.2 & 21.2 & -16.3 & / \\
\hline $9(2)$ & SPI $\cdots$ SPI & 11.56 & -10.5 & -3.2 & -15.8 & 19.2 & -15.4 & I \\
\hline $10(2)$ & SPI $\cdots$ SPI & 12.65 & -5.8 & -2.7 & -6.4 & 1.4 & -12.8 & I \\
\hline $11(1)$ & SPI $\cdots$ FA & 5.87 & -1 & -1.7 & -11.3 & 5.9 & -8.4 & / \\
\hline $12(1)$ & SPI $\cdots F A$ & 10.38 & -5.5 & -1.2 & -2.1 & 1 & -8.0 & / \\
\hline $13(1)$ & SPI $\cdots F A$ & 6.15 & -2.7 & -2.2 & -12.5 & 11.9 & -8.0 & I \\
\hline $14(1)$ & SPI $\cdots$ FA & 5.44 & -2.9 & -0.9 & -12.8 & 11.9 & -7.6 & / \\
\hline $15(1)$ & SPI $\cdots F A$ & 6.79 & -3.4 & -1.8 & -5.3 & 4.4 & -6.8 & I \\
\hline $16(1)$ & SPI $\cdots$ FA & 6.74 & -1.1 & -1.9 & -6.5 & 3 & -6.3 & / \\
\hline $17(1)$ & SPI $\cdots \mathrm{FA}$ & 9.38 & 0.5 & -2.6 & -5.6 & 4 & -3.8 & / \\
\hline $18(1)$ & SPI $\cdots \mathrm{FA}$ & 8.5 & 0.6 & -0.5 & -4.2 & 1.4 & -2.5 & I \\
\hline $19(1)$ & SPI $\cdots$ FA & 9.97 & 2.5 & -0.5 & -1.4 & 0.2 & 1.1 & I \\
\hline \multicolumn{9}{|c|}{$\mathrm{S}_{\mathrm{AA}}$} \\
\hline $1(2)$ & SPI $\cdots$ SPI & 5.99 & -14.1 & -5.2 & -73.5 & 46.2 & -54.2 & -55.0 \\
\hline $2(1)$ & SPI $\cdots$ AA & 6.83 & -50.5 & -12 & -16.6 & 52 & -44.7 & -47.4 \\
\hline $3(2)$ & SPI $\cdots$ SPI & 12.19 & -12.8 & -5.4 & -16.6 & 10.9 & -25.3 & -25.7 \\
\hline $4(2)$ & SPI $\cdots$ SPI & 12.59 & -10.8 & -4 & -14.9 & 10.1 & -21.1 & 1 \\
\hline $5(2)$ & SPI $\cdots$ SPI & 11.15 & -5.5 & -1.7 & -22.5 & 11.7 & -19.5 & I \\
\hline $6(1)$ & SPI $\cdots$ AA & 8.44 & -6.4 & -2.5 & -16.2 & 12.2 & -15.3 & 1 \\
\hline $7(2)$ & SPI $\cdots$ SPI & 9.36 & -8.2 & -2.7 & -16.4 & 18.9 & -13.2 & I \\
\hline $8(1)$ & SPI $\cdots$ AA & 6.59 & -3 & -0.6 & -15.1 & 8.4 & -11.6 & 1 \\
\hline $9(1)$ & SPI $\cdots$ AA & 6.47 & -0.6 & -0.8 & -12.6 & 6.6 & -8.1 & / \\
\hline
\end{tabular}




\begin{tabular}{|c|c|c|c|c|c|c|c|c|}
\hline 10(1) & SPI $\cdots$ AA & 6.95 & -1.3 & -1.1 & -7.8 & 2.2 & -7.6 & I \\
\hline 11(2) & SPI $\cdots$ SPI & 11.11 & -0.2 & -0.8 & -11.5 & 5.9 & -7.2 & 1 \\
\hline $12(2)$ & SPI $\cdots$ AA & 14.05 & -2.6 & -1.5 & -3.3 & 0.5 & -6.4 & I \\
\hline 13(2) & $\mathrm{AA} \cdots \mathrm{AA}$ & 5.99 & -2.3 & -0.7 & -1.8 & 0.5 & -4.3 & I \\
\hline 14(1) & SPI $\cdots$ AA & 8.54 & 1.5 & -1.1 & -5.7 & 2.4 & -2.6 & I \\
\hline \multicolumn{9}{|c|}{$\mathrm{S}_{\mathrm{Ben}}$} \\
\hline $1(2)$ & SPI $\cdots$ SPI & 6.18 & -14 & -4.8 & -64.2 & 36 & -52.0 & -53.4 \\
\hline $2(2)$ & SPI $\cdots$ SPI & 12.1 & -8.4 & -4 & -22.8 & 14.9 & -22.5 & -23.7 \\
\hline $3(2)$ & SPI $\cdots$ SPI & 12.62 & -7.4 & -5.5 & -17.1 & 12.9 & -18.8 & -19.9 \\
\hline $4(2)$ & SPI $\cdots$ SPI & 9.43 & -0.5 & -2.3 & -27.4 & 13.1 & -18.0 & 1 \\
\hline $5(1)$ & SPI $\cdots$ Ben & 7.12 & -5 & -2.1 & -22.5 & 14 & -17.8 & I \\
\hline $6(1)$ & SPI $\cdots$ Ben & 7.27 & -4.3 & -1.2 & -20.7 & 12.1 & -15.9 & I \\
\hline $7(2)$ & SPI $\cdots$ SPI & 12.78 & -8.2 & -4.3 & -9.2 & 10.1 & -13.7 & 1 \\
\hline $8(1)$ & SPI $\cdots$ Ben & 7.29 & -3.2 & -1.2 & -14 & 7.6 & -11.7 & 1 \\
\hline $9(1)$ & SPI $\cdots$ Ben & 9.44 & -6.8 & -2.6 & -6.5 & 10.5 & -8.4 & I \\
\hline $10(1)$ & SPI $\cdots$ Ben & 7.26 & -0.9 & -1 & -11.9 & 6 & -8.3 & I \\
\hline 11(1) & SPI $\cdots$ Ben & 6.99 & -0.3 & -0.4 & -11.3 & 4.9 & -7.4 & I \\
\hline 12(2) & SPI $\cdots$ SPI & 11.09 & 0.8 & -0.8 & -9.9 & 3.4 & -6.3 & I \\
\hline 13(2) & Ben $\cdots$ Ben & 5.96 & -1.4 & -0.2 & -7.3 & 3.6 & -5.7 & 1 \\
\hline $14(2)$ & Ben $\cdots$ Ben & 6.18 & -1.6 & -0.2 & -6.7 & 3.6 & -5.4 & I \\
\hline \multicolumn{9}{|c|}{$\mathrm{S}_{\mathrm{Tol}}$} \\
\hline $1(2)$ & SPI $\cdots$ SPI & 6.04 & -12.9 & -5.3 & -71 & 43 & -52.8 & -52.4 \\
\hline $2(1)$ & SPI $\cdots$ Tol & 5.69 & -7.8 & -2.1 & -37.3 & 22.4 & -28.4 & -29.8 \\
\hline $3(1)$ & SPI $\cdots$ Tol & 6.49 & -7.5 & -1.6 & -33.3 & 22.5 & -24.2 & -26.2 \\
\hline $4(2)$ & SPI $\cdots$ SPI & 12.1 & -13.7 & -5.9 & -15.4 & 16.7 & -21.9 & 1 \\
\hline $5(2)$ & SPI $\cdots$ SPI & 12.33 & -9.1 & -3.4 & -19.5 & 12.8 & -21.2 & I \\
\hline $6(2)$ & SPI $\cdots$ SPI & 10.94 & -8.5 & -1.7 & -16.5 & 12.4 & -16.9 & I \\
\hline $7(2)$ & SPI $\cdots$ SPI & 12.17 & -7 & -2.9 & -8 & 4.4 & -13.8 & / \\
\hline $8(1)$ & SPI $\cdots$ Tol & 6.69 & -1.7 & -0.5 & -17.3 & 9.3 & -11.6 & 1 \\
\hline $9(1)$ & SPI $\cdots$ Tol & 9.62 & -2.4 & -2.6 & -8.6 & 7 & -7.7 & I \\
\hline $10(1)$ & SPI $\cdots$ Tol & 7.48 & -0.4 & -0.2 & -11.8 & 5.2 & -7.6 & I \\
\hline 11(2) & Tol $\cdots$ Tol & 6.04 & -1.7 & -0.2 & -8.7 & 5.1 & -6.4 & I \\
\hline $12(2)$ & SPI $\cdots$ SPI & 11.17 & 2.4 & -0.3 & -4.8 & 0.2 & -1.8 & / \\
\hline $13(1)$ & SPI $\cdots$ Tol & 10.06 & 0.5 & -0.1 & -2.2 & 0.1 & -1.4 & I \\
\hline
\end{tabular}




\begin{tabular}{|c|c|c|c|c|c|c|c|c|}
\hline \multicolumn{9}{|c|}{$\mathrm{S}_{\mathrm{ACN} \text {-new }}$} \\
\hline $1(2)$ & SPI $\cdots$ SPI & 11.11 & -12.6 & -5.5 & -25.2 & 10.7 & -32.8 & -35.3 \\
\hline $2(2)$ & SPI $\cdots$ SPI & 8.11 & -2.5 & -4.5 & -34.7 & 15.9 & -26.4 & -28.7 \\
\hline $3(1)$ & SPI $\cdots$ ACN & 6.57 & -15.3 & -4.2 & -15.7 & 12.3 & -25.4 & -26.7 \\
\hline $4(2)$ & SPI $\cdots$ SPI & 9.69 & -8.6 & -4.5 & -21.4 & 13.2 & -22.9 & I \\
\hline $5(2)$ & SPI $\cdots$ SPI & 9.92 & -2 & -3.3 & -24.8 & 10.2 & -19.8 & I \\
\hline $6(1)$ & SPI $\cdots$ ACN & 5.79 & -11.5 & -5.1 & -10.2 & 8.9 & -19.3 & I \\
\hline $7(1)$ & SPI $\cdots$ ACN & 8.47 & -16.2 & -3.9 & -10.9 & 16.7 & -19.2 & / \\
\hline $8(1)$ & SPI $\cdots$ ACN & 8.04 & -13.6 & -2.4 & -9.2 & 12.2 & -16.6 & / \\
\hline $9(2)$ & SPI $\cdots$ SPI & 9.61 & -0.9 & -1 & -20.9 & 9.6 & -14.1 & 1 \\
\hline $10(2)$ & SPI $\cdots$ SPI & 10.24 & -2.5 & -1.9 & -15.8 & 9 & -12.2 & 1 \\
\hline 11(1) & SPI $\cdots$ ACN & 5.66 & 0.1 & -1.8 & -12.5 & 8.1 & -7.1 & I \\
\hline 12(1) & SPI $\cdots$ ACN & 7.02 & 2.5 & -1.5 & -9.4 & 5.1 & -3.4 & I \\
\hline \multicolumn{9}{|c|}{ Form I } \\
\hline $1(2)$ & SPI $\cdots$ SPI & 6.23 & -11.1 & -4.8 & -56.7 & 26.5 & -48.3 & -50.5 \\
\hline $2(2)$ & SPI $\cdots$ SPI & 9.82 & -6.6 & -3.1 & -42.6 & 26.9 & -29.7 & -31.7 \\
\hline $3(2)$ & SPI $\cdots$ SPI & 10.67 & -11.4 & -5.9 & -21 & 14.4 & -25.8 & -27.0 \\
\hline $4(2)$ & SPI $\cdots$ SPI & 10.13 & -2.8 & -3.8 & -31.5 & 19.8 & -21 & 1 \\
\hline $5(2)$ & SPI $\cdots$ SPI & 9.98 & -4.1 & -2.2 & -15.4 & 6.4 & -15.4 & 1 \\
\hline $6(2)$ & SPI $\cdots$ SPI & 11.76 & -6.8 & -2.4 & -12.3 & 7.4 & -15.2 & 1 \\
\hline $7(2)$ & SPI $\cdots$ SPI & 15.32 & -1.1 & -2.3 & -4.5 & 3.9 & -4.3 & / \\
\hline \multicolumn{9}{|c|}{ Form II } \\
\hline $1(2)$ & SPI $\cdots$ SPI & 6.35 & -10.9 & -5.6 & -54.7 & 33.1 & -42.8 & -44.9 \\
\hline $2(2)$ & SPI $\cdots$ SPI & 10.46 & -15.2 & -6 & -33.3 & 22.5 & -35.6 & -37.4 \\
\hline $3(2)$ & SPI $\cdots$ SPI & 9.87 & -9 & -6.8 & -35.1 & 16.7 & -34.9 & -36.4 \\
\hline $4(2)$ & SPI $\cdots$ SPI & 12.36 & -10.8 & -3.5 & -13.2 & 6.6 & -21.3 & 1 \\
\hline $5(2)$ & SPI $\cdots$ SPI & 11.95 & -10.6 & -2.8 & -14.1 & 11.4 & -18.6 & / \\
\hline $6(2)$ & SPI $\cdots$ SPI & 11.01 & 0.8 & -0.9 & -14.2 & 8.3 & -7.1 & I \\
\hline $7(2)$ & SPI $\cdots$ SPI & 9.55 & 4.3 & -0.7 & -11.3 & 1.2 & -5.1 & I \\
\hline
\end{tabular}

${ }^{a} \mathrm{All}$ the energy values are in $\mathrm{kJ} / \mathrm{mol}$. ${ }^{\mathrm{b}}$ The symbol of $\mathrm{R}_{\mathrm{dist}}$ is the distance between molecular centroids ${ }^{\mathrm{c}}$ Electrostatic $\left(E_{\text {ele }}\right)$, polarisation $\left(E_{\mathrm{pol}}\right)$, dispersion $\left(E_{\mathrm{dis}}\right)$ and exchange-repulsion energy $\left(E_{\text {rep }}\right)$ contributions. ${ }^{1-3}$ 

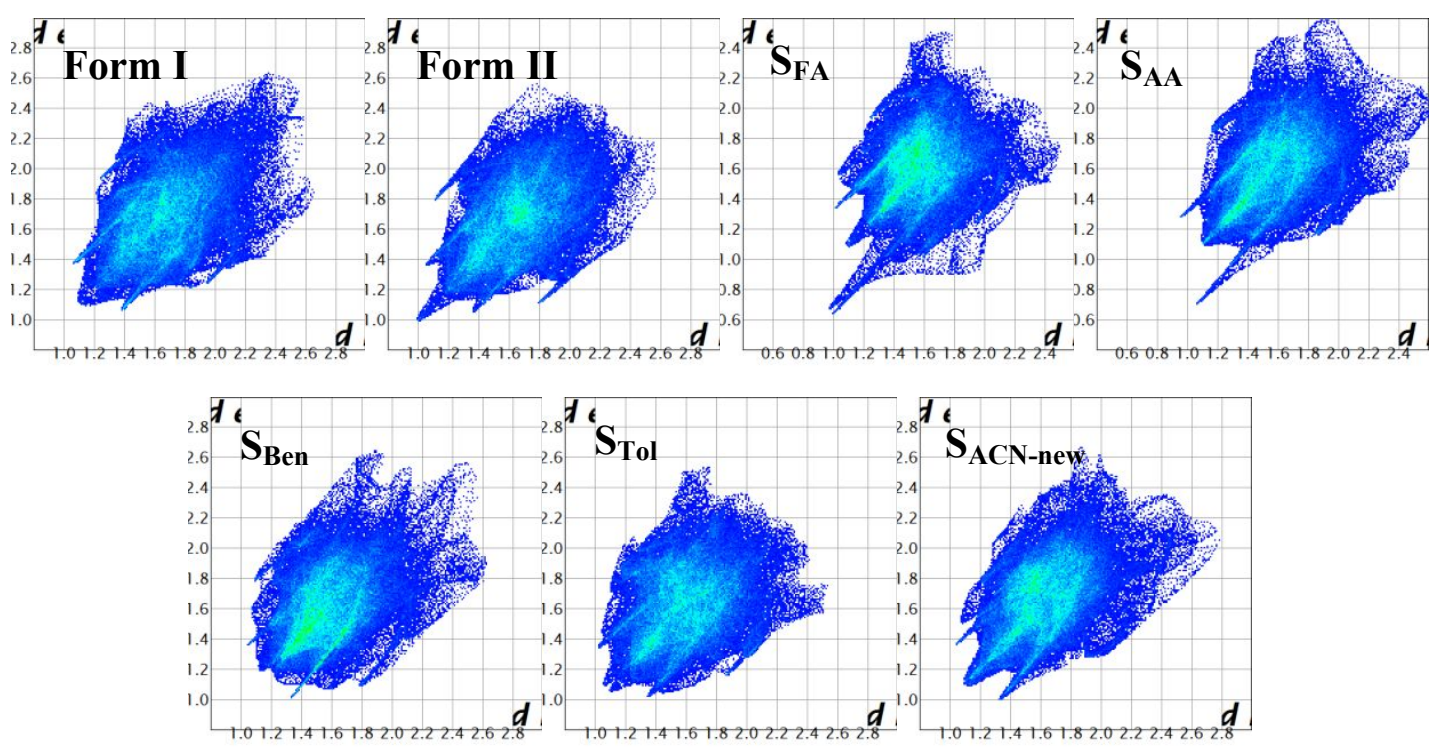

Figure S9 2D fingerprint plots of Hirshfeld surfaces for SPI molecule in different forms

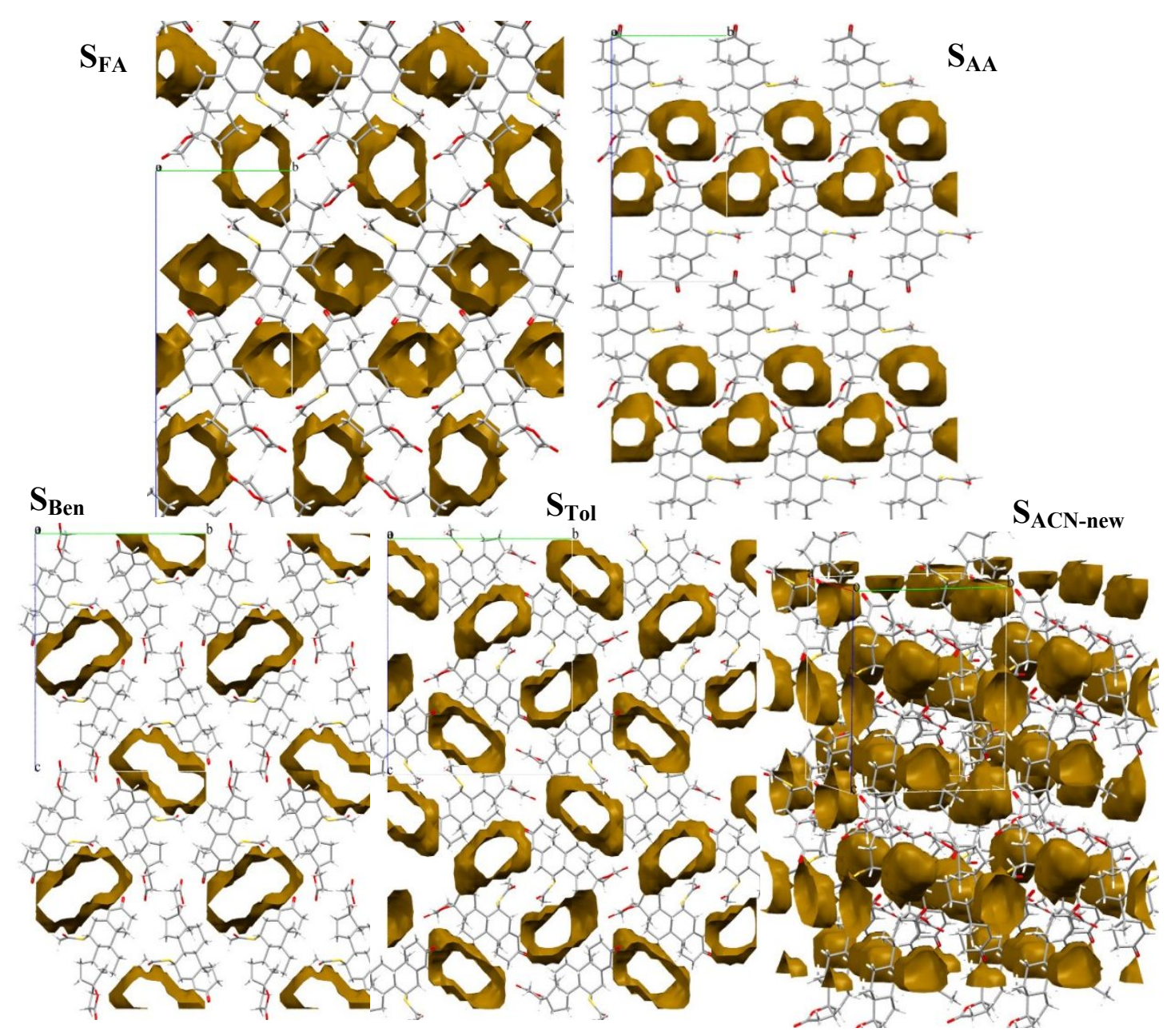

Figure S10 Void maps in SPI solvate framework structure after removing the solvent molecules.

The void maps were calculated by Mercury (2020.1). The conditions were set as follows: (a) probe radius $1.2 \AA$, approximately grid spacing $0.7 \AA$, calculated using the contact surface. 

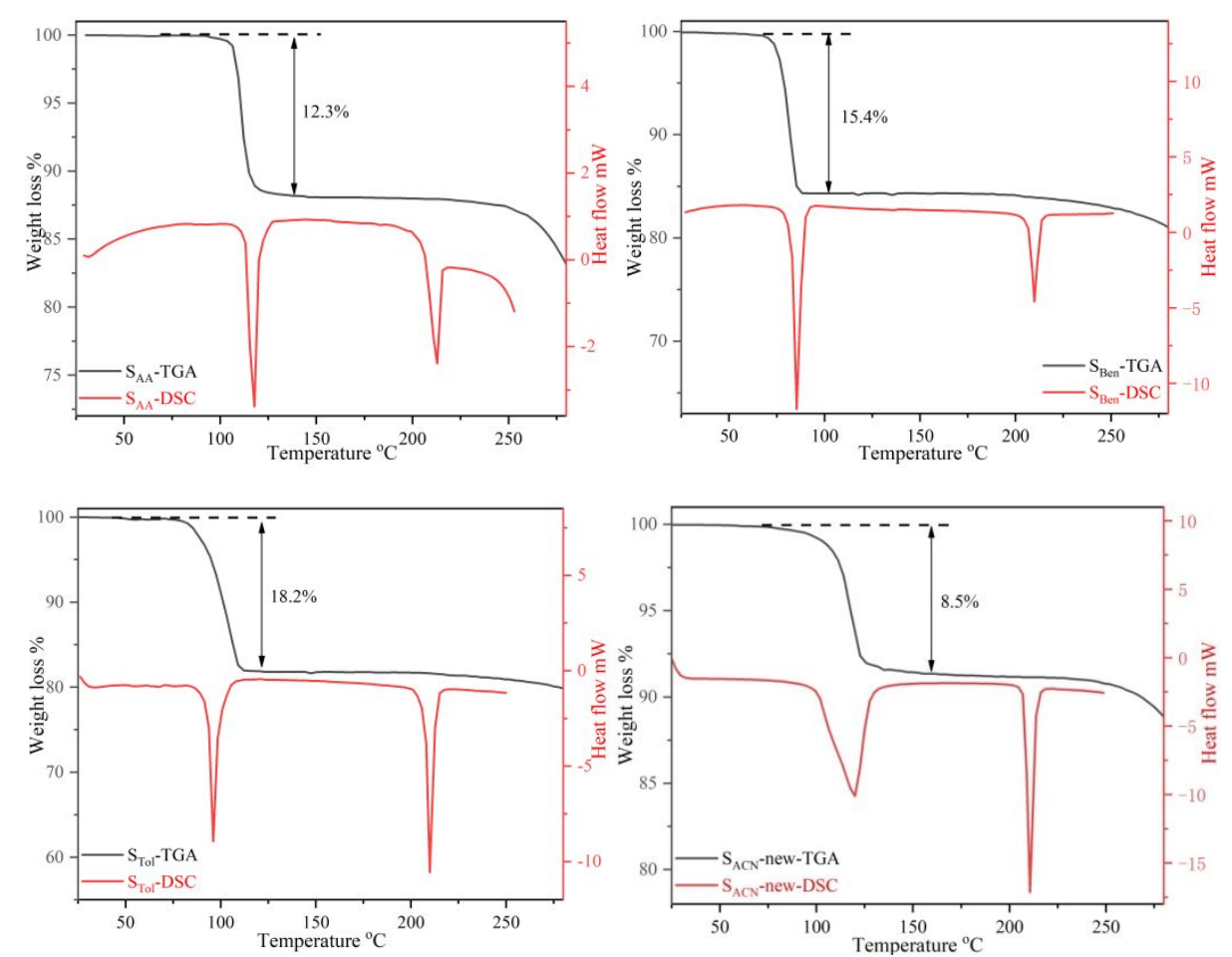

Figure S11 Overlaid DSC and TGA profiles of SPI solvate.
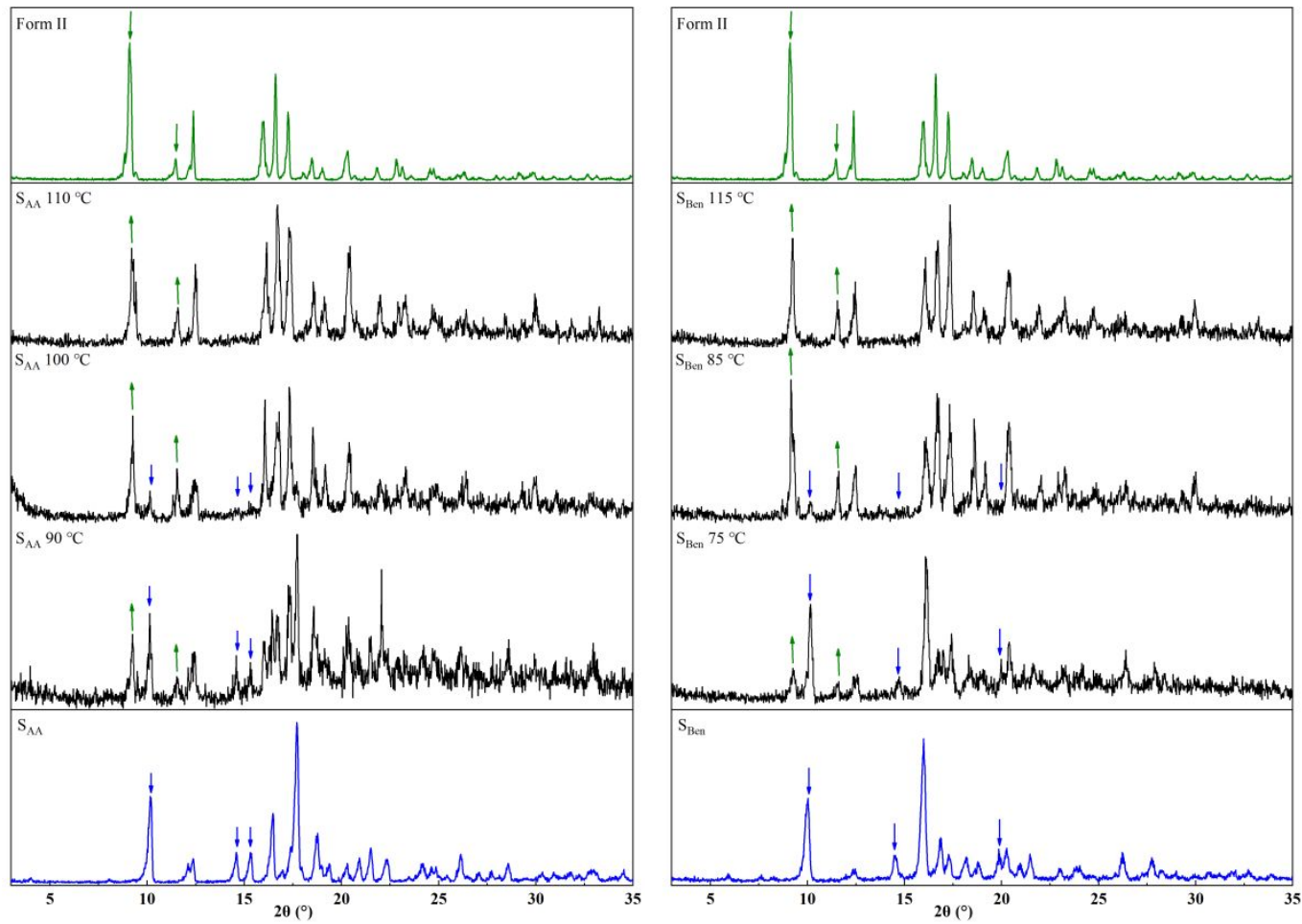

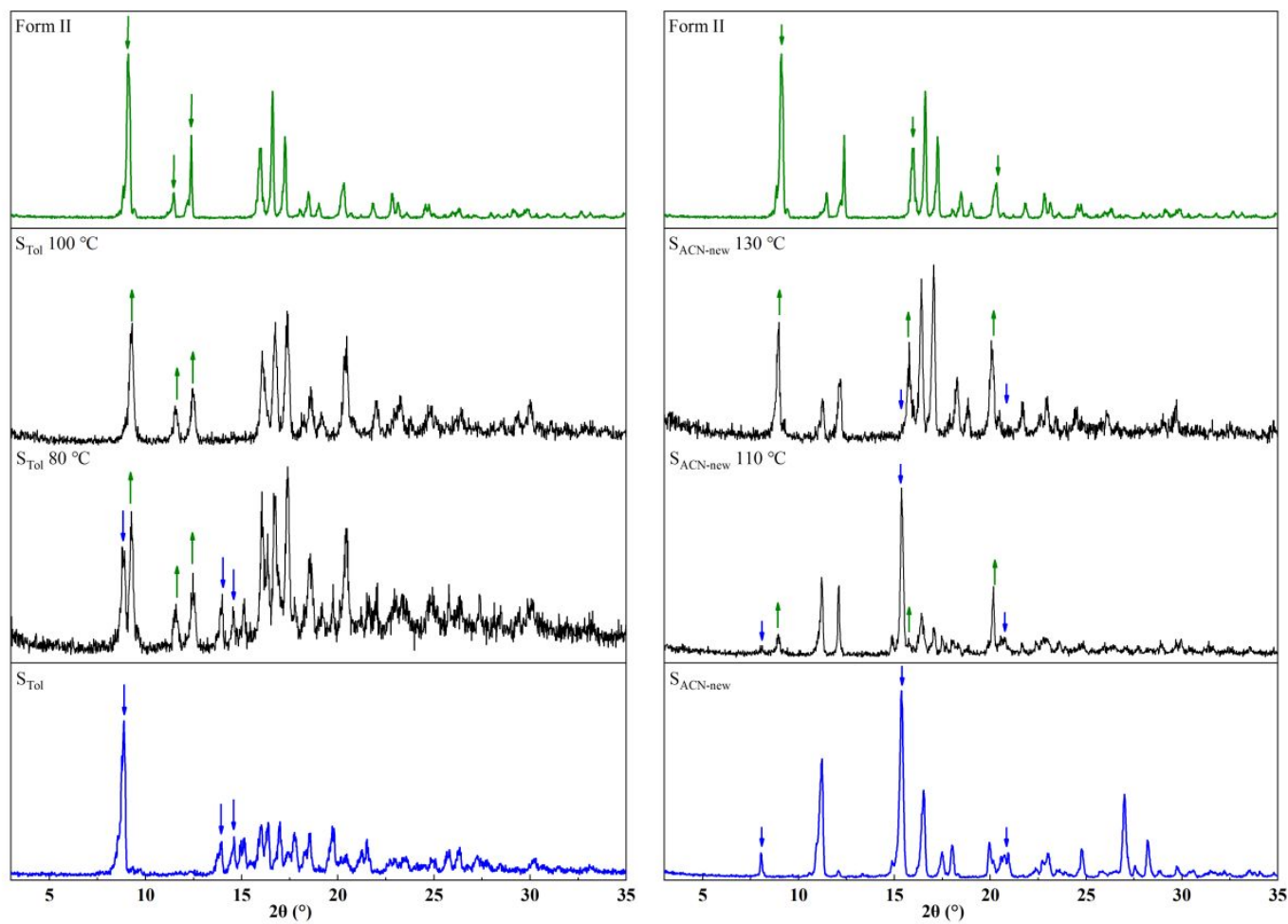

Figure S12 The desolvation and phase transformation process of different SPI solvates under variable temperature (VT-PXRD).

(a) $\mathrm{S}_{\mathrm{AA}}$

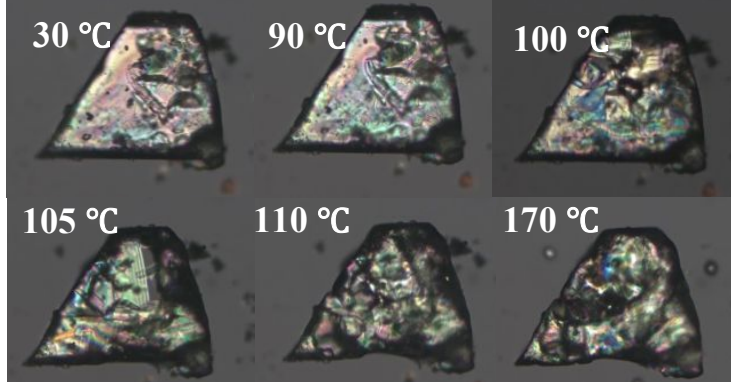

(b) $\mathbf{S}_{\text {Ben }}$
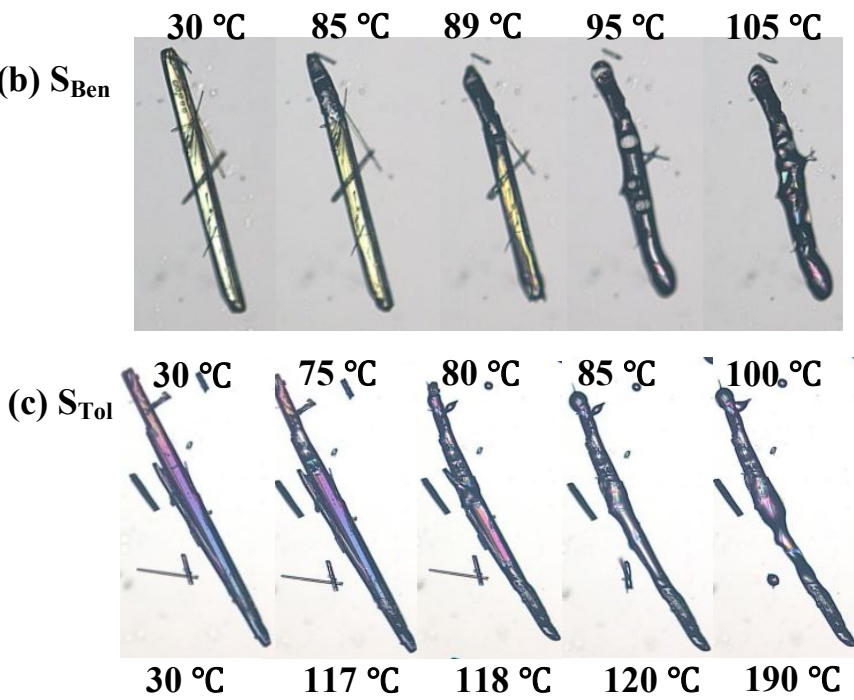
(d) $\mathrm{S}_{\mathrm{ACN}}$

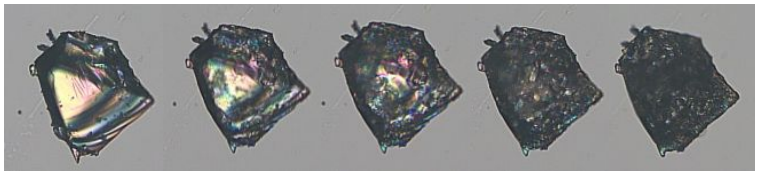

Figure S13. Hot stage microscopy (HSM) images of SPI solvates during the desolvation process.

The effect of the structure determination temperature on the calculated packing index was evaluated by using structure determination in different temperatures. Similar results (packing index 0.661 and void space of $0 \%$ ) were obtained for $\mathrm{S}_{\mathrm{AA}}$ determined at 113 and $295 \mathrm{~K}$ (Table S3).

The results showed that, in general, cell shrinkage resulting from the measurements in lower temperature $(113 \mathrm{~K})$ reduces the packing index by $\sim 0.025$.

Table S3 Crystallographic data for SPI Forms

\begin{tabular}{cc}
\hline & $\mathrm{S}_{\mathrm{AA}}$ \\
\hline Formula & $\mathrm{C}_{24} \mathrm{H}_{32} \mathrm{O}_{4} \mathrm{~S} \cdot \mathrm{C}_{2} \mathrm{H}_{4} \mathrm{O}_{2}$ \\
Formula weight & 476.61 \\
Crystal system & Monoclinic \\
Space group & $P 2_{1}$ \\
Temperature $(\mathrm{K})$ & 293 \\
$a(\AA)$ & $6.0578(12)$ \\
$b(\AA)$ & $9.4403(19)$ \\
$c(\AA)$ & $21.896(4)$ \\
$\alpha\left({ }^{\circ}\right)$ & 90 \\
$\beta\left({ }^{\circ}\right)$ & 91.96 \\
$\gamma\left({ }^{\circ}\right)$ & 90 \\
Cell volume $\left(\AA^{3}\right)$ & 1219.2 \\
$\left.\rho_{\text {calc. }}(\mathrm{g} / \mathrm{cm})^{3}\right)$ & 1.298 \\
$\mathrm{Z}$ & 2 \\
$R$ & 0.0453 \\
$R_{1}(\mathrm{I}>2 \sigma(\mathrm{I}))$ & 0.0453 \\
$w R_{2}$ & 0.113 \\
$\mathrm{GOF}(\mathrm{S})$ & 1.006 \\
$\mathrm{CCDC}$ & 2038599 \\
\hline
\end{tabular}

\section{Reference}


(1) Turner, M. J.; Grabowsky, S.; Jayatilaka, D.; Spackman, M. A. Accurate and Efficient Model Energies for Exploring Intermolecular Interactions in Molecular Crystals. J Phys Chem Lett 2014, 5, 4249-55.

(2) Turner, M. J.; Thomas, S. P.; Shi, M. W.; Jayatilaka, D.; Spackman, M. A. Energy Frameworks: Insights into Interaction Anisotropy and the Mechanical Properties of Molecular Crystals. Chem. Commun. 2015, 51, 3735-8.

(3) Mackenzie, C. F.; Spackman, P. R.; Jayatilaka, D.; Spackman, M. A. CrystalExplorer Model Energies and Energy Frameworks: Extension to Metal Coordination Compounds, Organic Salts, Solvates and Open-shell Systems. IUCrJ. 2017, 4, 575-587. 